\title{
Notas para la comprensión del valor de uso en la concepción de Bolívar Echeverría
}

\section{JOSÉ RAMÓN CARMONA MOTOLINÍA*}

En un afán por profundizar y extender la crítica sobre la modernidad capitalista, el texto analiza la función de la categoría valor de uso. El filósofo Bolívar Echeverría caracteriza y reconstruye esta categoría para plantear en términos teóricos los elementos involucrados en la configuración histórica de la forma social-natural. En ese sentido, se proponen algunas notas para comprender, desde la perspectiva de Echeverría, los mecanismos que permiten la subordinación o subsunción del «valor de uso concreto» al «valor de cambio abstracto».

*Estudiante

del doctorado en la Unidad

Académica de Estudios del

Desarrollo,

Universidad

Autónoma de

Zacatecas

\section{Introducción}

La finalidad de este ensayo es identificar elementos que componen la categoría valor de uso, desde la concepción teórica de Bolívar Echeverría, para comprender su separación y subordinación en el plano objetivo-material al valor de cambio en el modo de reproducción social capitalista. Aunque la idea misma de valor de uso es extraída del discurso teórico crítico de Marx, Echeverría propone una reconstrucción de la misma.

El análisis parte de una revisión teórica-conceptual de la obra de Bolívar Echeverría, en particular de algunos textos en los que se aborda la categoría. En ese marco se plantea que ésta posee un carácter explicativo central dentro de su discurso teórico y constituye una herramienta clave para el pensamiento crítico.

\section{Planteamientos de Bolívar Echeverría en torno al valor de uso}

En principio, habría que cuestionar el papel que desempeña en el discurso teórico de Bolívar Echeverría la categoría de valor de uso. Sobresale la discusión correspondiente a su significado, por lo que se alude a la intención de Echeverría por recuperarlo. Un primer planteamiento se expone en Definición de la cultura, donde se identifican ciertos elementos que actúan en el proceso de reproducción de la vida humana. Si bien no opera directamente, la categoría se emplea para problematizar la definición actual de la cultura como concepto desarrollado en diferentes discursos teóricos; incluso se intenta recuperar los aspectos relativos a la forma natural que se encuentran en ciertas posturas, como la estructuralista y la existencialista.

Al respecto, se parte de una caracterización de la lógica de reproducción social como autoproducción, la cual se explica por el 
hecho de que el ser humano no es sólo un ser natural, a pesar de pertenecer a la «naturaleza» como animal. De ese modo, al producirse y reproducirse a sí mismo, el ser humano tiene necesidad de interactuar con lo Otro (la naturaleza), a partir de los objetos prácticos que extrae para realizar sus acciones cotidianas, en preocupación constante por el «horizonte de escasez». Estos objetos prácticos son, según Echeverría, «productos con valor de uso» y se diferencian de aquellos «objetos intermedios» que aparecen en los procesos de reproducción animal. La diferencia radica en que,

el bien producido para el disfrute humano y por el trabajo humano es un objeto muy especial: su figura concreta, no informe, no es la de un ejemplar más de una «figura» general (o abstracta), indefinidamente repetida, como es la de los «objetos» animales; ella es formada $\mathrm{y}$, por tanto, en principio, singular (o concreta), única e irrepetible. ${ }^{1}$

Dentro de dicha conceptualización se expone uno de los aspectos fundamentales acerca del proceso de reproducción del sujeto social: se da forma a sí mismo en un proceso de realización. En tal proceso ocurre una primera fase de producción y la posterior entraña el consumo o disfrute. La reconfiguración de esta forma del sujeto social se da en la medida en que «al abrir un horizonte de posibilidades de forma» para los objetos de consumo, también se abre otro para la producción y la reproducción: «En la fase productiva, el sujeto humano proyecta convertirse a sí mismo en alguien diferente, adquirir otra forma; después, en la fase consuntiva, al asumir dicha forma, lleva a cabo ese proyecto». ${ }^{2}$

Desde este enfoque se concibeel elemento de la «forma natural», así como los valores de uso que constituyen los objetos de la materia (natu-

${ }^{1}$ Bolívar Echeverría, Definición de la cultura (segunda edición), México, Fondo de Cultura Económica, 2010, p. 64. ${ }^{2}$ Ibid, p. 73. raleza), para transformarlos en «objetos prácticos» al alcance del sujeto humano socialmente construido. No obstante, la forma natural no se concibe como una «substancia» o «naturaleza humana» atemporal; por el contrario, se refiere a la cualidad del sujeto que construye su propia forma histórica, una «deformación» en el «acto de trans-naturalización»:

La «forma natural» de la vida humana — del proceso de reproducción de sí misma y del mundo en que se desenvuelve - es una forma social e histórica; es el modo que tiene el ser humano de auto-afirmarse e identificarse mientras se define o se determina en referencia a lo otro, a la «naturaleza». Es la forma «meta-física» que adoptan las funciones «físicas» o vitales del animal humano cuando éste comienza a ejercer una sujetidad, esto es, a ser «libre». ${ }^{3}$

Ahora bien, en un segundo planteamiento, al analizar los procesos histórico-sociales por medio de los cuales se configura el proyecto de la modernidad capitalista, Echeverría refiere el lugar que ocupan elementos conceptuales mencionados en la argumentación previa. Con base en las proposiciones expuestas, el valor de uso sería una de las «dos lógicas» o «principios estructuradores» contradictorios, inherentes a la sociedad capitalista: por un lado, la del valor de uso «cualitativo o social-natural»; y, por otro, la forma de valor que lo subordina como un fin en sí mismo y se autonomiza. ${ }^{4}$

Lógica o principio estructurador alude a un comportamiento de carácter económico que organiza las prácticas en la producción y reproducción material del sujeto social. Así, el desarrollo de la categoría valor de uso es un aporte a

\footnotetext{
${ }^{3}$ Bolívar Echeverría, La «modernidad americana» (claves para su comprensión), México, Universidad Nacional Autónoma de México, 2007, p. 23.

${ }^{4}$ Bolívar Echeverría, Valor de uso y utopía, Mexico, Siglo XXI, 1998.
} 
la reconstrucción de la concepción de la «forma natural» de objetos o cosas como valores de uso, una contribución a la teoría del valor de Marx.

La categoría de valor de uso refiere a la utilidad de lo Otro (la naturaleza), que el sujeto social reproduce como objeto práctico en la fase de producción y en la de consumo. Del mismo modo que en el proceso de producción de objetos prácticos, en los de acción e interacción del sujeto humano con su entorno existe una mediación, debido a la existencia de valores de uso. Esto significa que el valor de uso es una mediación por la cual el sujeto social se plantea a sí mismo su forma y durante el consumo del objeto se proyecta, se transforma y autoidentifica.

Echeverría asume la posibilidad concreta del sujeto de elegir su forma en libertad (la forma natural), que se configura desde los valores de uso, de ahí su trascendencia. El valor de uso permite la configuración de la capacidad histórica del sujeto humano de darse forma, en relación con los elementos de carácter práctico que produce y consume. De esa manera, esta capacidad puede representarse y expresarse en diferentes formas y modos, en cuanto los valores de uso son diversos, adecuados a las condiciones de producción, reproducción y consumo del sujeto social. Esta perspectiva de elegirse - la cual sucede con la forma natural y los valores de uso - establece el marco para considerar el surgimiento de formas sociales e históricas cualitativamente diferentes del sujeto humano.

Sin embargo, dentro de la configuración histórica del modo de reproducción social capitalista la forma de valor subordina a la forma natural y el valor de uso depende del valor de cambio, de la forma mercancía de los objetos que se valorizan.

La vida humana en su «forma de valor» es como un «doble» o un «fantasma» de lo que es ella misma en su «forma natural»; es una proyección objetivada de su propio proceso de reproducción en lo que él tiene, entre otras cosas, de capacidad de creación y destrucción de valor económico dentro del mundo de las mercancías capitalistas o, lo que es lo mismo, en lo que él tiene, abstractamente, de vehículo suficiente para el proceso de autovalorización del valor capitalista o proceso de acumulación de capital. ${ }^{5}$

Aquí opera el principio estructurador de la forma valor, dado que la vida social se organiza por un proceso en el que el ser humano sólo actúa en calidad de fuerza de trabajo. El valor de uso se somete a la lógica en la que todo lo que se produce y consume en la sociedad moderna debe orientarse a reproducir el proceso de acumulación de capital. Los valores de uso funcionan en razón de dicho proceso. Es decir, los objetos prácticos (derivados de la «naturaleza») que se producen y consumen sólo tienen sentido como portadores de valor de cambio (abstracto); mientras que en el plano de la interacción social únicamente se lleva a cabo mediante el proceso de intercambio de mercancías, cada una de las cuales es portadora de un valor de uso.

En ese sentido se expresa el fenómeno de enajenación, que consiste en la producción de mercancías consumidas en la sociedad capitalista moderna, sin que actúe la mediación del valor de uso por estar subordinada. En tanto enajenación, la sujetidad de esa vida y su capacidad política de autoproducirse, autoidentificarse y elegirse, es sustituida por el valor económico que plantea sus propios requerimientos de autovalorización sobre los de la forma social-natural.

\section{La forma natural y el valor de uso: elementos para su comprensión}

Desde esta conceptualización, Echeverría se propone comprender la ruptura que ocurre en el proceso de producción/consumo y de

5 Bolívar Echeverría, La «modernidad americana»..., pp. 24-25. 
reproducción de la forma social-natural. La existencia en ruptura es una cuestión que se origina por la cultura y permite al sujeto humano la adopción de diversas formas. A partir de la diversidad de las configuraciones históricas, esa capacidad creadora —inscrita en la producción de objetos prácticos con valor de uso como utilidad de lo Otro (la naturaleza) - forja comportamientos que el sujeto reproduce en libertad al convertirse en uno de los ejes que ofrece la oportunidad de moldear las diferentes maneras de existencia, las cuales en determinado momento pueden configurarse como modos de vida o estrategias de subsistencia.

La forma valor establecida con la sociedad capitalista moderna constituye una posibilidad abierta, en ese amplio marco de formas sociales históricas. ${ }^{6}$ Debido a que el valor de uso depende de la lógica de la forma valor, también se limita su capacidad de brindar una configuración distinta de la forma del sujeto social. No obstante, dicha limitación no sucede por la lógica misma del valor de uso, que se sujeta a la contradicción provocada por la estructuración derivada del valor de cambio, sino que su capacidad es anulada y queda encerrada la posibilidad creadora e inventora de otras formas en la lógica del valor de cambio.

En ese contexto, el proceso de autovalorización inherente a la configuración histórica del modo de producción y reproducción capitalista se construye sobre esos elementos; en palabras de Eche-

${ }^{6}$ Bolívar Echeverría, Las ilusiones de la modernidad: ensayos, 1, México, El Equilibrista, 1995. verría, encubre estos aspectos del proceso de realización. La comprensión del valor de uso tiene una importancia fundamental para pensar las posibilidades del sujeto humano de elegirse, desde una forma social-natural inserta en sus mismas capacidades de autoproducción. Ello comprende la potencialidad para producir formas cualitativamente distintas, en un horizonte donde la sujetidad está reproducida en los diversos valores de uso creados por variadas formas sociales.
La comprensión del valor de uso tiene una importancia fundamental para pensar las posibilidades del sujeto humano de elegirse, desde una forma socialnatural inserta en sus mismas capacidades de autoproducción.

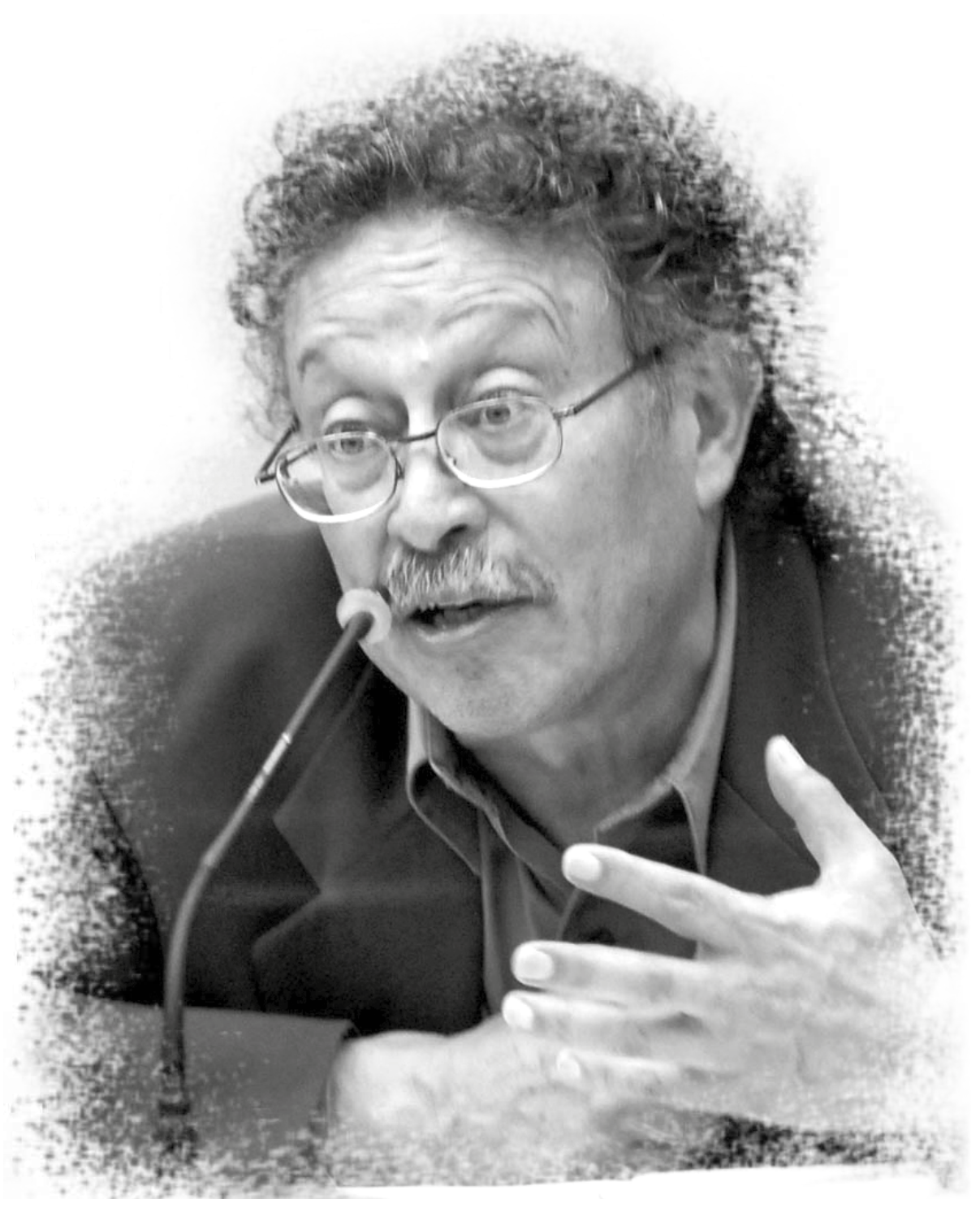


¿Quién construyó Tebas, la de las Siete Puertas?

En los libros aparecen los nombres de los reyes.

¿Arrastraron los reyes los bloques de piedra?

Y Babilonia, destruida tantas veces,

¿quién la volvió siempre a construir? ¿En qué casas de la dorada Lima vivían los constructores?

¿A dónde fueron los albañiles la noche en que fue terminada la Muralla China? La gran Roma está llena de arcos de triunfo. ¿Quién los erigió?

¿Sobre quiénes

triunfaron los Césares? ¿Es que Bizancio, la tan cantada, sólo tenía palacios para sus habitantes? Hasta en la legendaria Atlántida,

la noche en que el mar se la tragaba, los que se hundían gritaban llamando a sus esclavos.

El joven Alejandro conquistó la India. ¿Él solo?
César derrotó a los galos.

¿No llevaba siquiera cocinero?

Felipe de España lloró cuando su flota

fue hundida. ¿No lloró nadie más?

Federico II venció en la Guerra de los Siete Años.

¿Quién

venció además de él?

Cada página una victoria.

¿Quién cocinó el banquete de la victoria?

Cada diez años un gran hombre.

¿Quién pagó los gastos?

Tantas historias.

Tantas preguntas.
Preguntas de un obrero que lee Bertolt Brecht

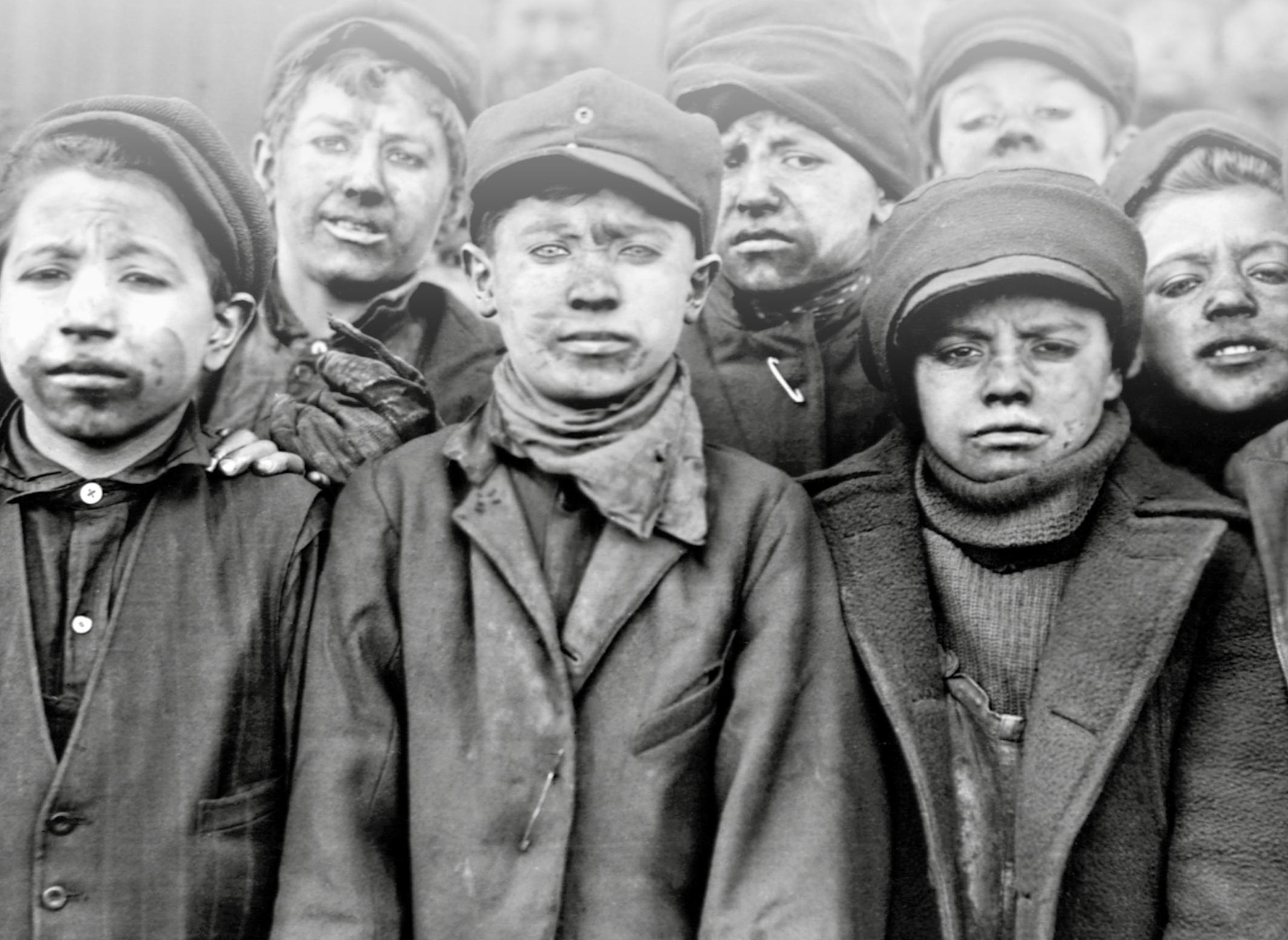

